\title{
THE PLACE OF ACCURATE PROPER MOTIONS IN GALACTIC RESEARCH
}

\author{
A. BLAAUW
}

Problems in galactic research for the solution of which highly accurate absolute proper motions are required are manyfold. The following selection seems of particular interest at the present moment. Problems for which only accurate relative proper motions are required are not considered here.

(a) Objects exhibiting spiral structure (OB stars, early-type supergiants, luminous Cepheids, youngest open clusters).

Particular reference is made to the problem of the local deviations from circular motion; 'local' implying regions with dimensions of $100-1000$ parsecs. Their study is of great interest for the problem of the origin of the spiral structure. Assuming that the youngest stars, ages up to several million years, still possess the kinematical properties of the interstellar medium, these stars may be expected to reveal the regional kinematical characteristics of the gas. $21-\mathrm{cm}$ observations have clearly indicated the existence of deviations from circular motions up to about $-30 \mathrm{~km} / \mathrm{sec}$. These, however, refer to the line-of-sight component only and the distances of the objects concerned are uncertain.

Velocities of the order of $10-30 \mathrm{~km} / \mathrm{sec}$ correspond to 0.002 to $0 " 006$ at a distance of 1000 parsec. Hence, if an absolute accuracy can be obtained of about $0^{\prime \prime} 002$, important information on regional kinematics may be obtained up to 2 or $3 \mathrm{kpc}$.

For somewhat older stars, whose motions may not any more exhibit the characteristics of the interstellar medium, accurate proper-motion measurements may throw light on the possible existence of a systematic lag of the mean gas motion with respect to the circular motion in a gravitational field.

For these reasons, continued observations of the list of $\mathrm{O}$ and $\mathrm{B}$ stars North of $-20^{\circ}$ declination and brighter than 8.5 visual magnitude, proposed in the IAU Symposium Report No. 1 (1953) for meridian observations appear highly desirable. Exploratory use of recent observations of these stars shows that new proper motions of high accuracy may be obtained. Extension of this list is strongly recommended.

High-precision proper motions of the stars mentioned may also throw important light on possible systematic errors in the fundamental system through the analysis of the proper-motion components perpendicular to the galactic plane.

Perek (ed.), Highlights of Astronomy, 316-318. @ I.A.U. 
(b) Bright stars of spectral types $A$ and $F$ for tracing of past orbit and location of origin.

Strömgren and associates have demonstrated the possibility of locating the region of origin of stars of ages up to a few hundred million years. Application of the method depends to a large extent on the accuracy of the proper motions. For a 6 magnitude A-type star, at about 100 parsecs, a probable error of 0.002 corresponds to $1 \mathrm{~km} / \mathrm{sec}$; this accuracy is just about sufficient. For fainter, more distant stars the permissible probable errors must be correspondingly reduced.

(c) Bright stars that served as reference stars in the McCormick faint star programmes.

The McCormick Observatory has published large numbers of proper motions of faint stars in about 700 regions North of $-30^{\circ}$ declination, centered on bright stars. The first epoch plates were taken for the determination of the trigonometric parallaxes of these stars.

Meridian observations and, thereby, high-precision fundamental proper motions of these stars are desirable for two reasons:

(1) These stars are used to reduce the proper motions of the faint stars to the absolute system. Studies of the systematic motions of the faint stars have proved very useful in recent analyses for the redetermination of the constants of precession and galactic rotation.

(2) Studies of the absolute proper motions of the faint stars in the McCormick fields. Of particular interest will be the systematic and peculiar motions of the low metal content late-type giants, discriminated photometrically (disk population), which may be assumed to have a smooth density distribution and smoothed-out distribution of velocities. The solar motion with respect to these stars should provide important and accurate information on the component of the Sun's motion with respect to the galactic centre, and hence on this component of the motion of the very young population in the solar neighbourhood.

This programme of bright stars should be carried out in conjunction with the extensive current programme of photographic redetermination of the proper motions of the faint stars in the McCormick fields.

(d) The Hyades.

Recent reconsideration of the distance scale has emphasized the importance of the role of the Hyades. Continued observations of the Hyades stars is therefore most desirable, with particular attention to the outlying members.

\section{DISCUSSION}

W. Dieckvoss: The AGK3 preliminary catalogue contains about $3500 \mathrm{M}$-type stars fainter than magnitude 10 and with proper motions known with a mean error of $\pm 0 \% 008$ per year. Are these stars likely to be useful in the study of the old disk population? 
A. Blaauw: Yes, especially if accurate spectroscopic data become available, for then they could be used to determine the solar motion with respect to the galactic centre.

C.A. Murray: The McCormick material discussed by Williams and Vyssotsky does not cover the complete sphere. I feel that the McCormick work should be extended to the Southern hemisphere by utilising the magnitude compensated parallax fields at Southern observatories like Yale and Cape. 